\title{
Conductance through glycine in a graphene nanogap
}

\author{
Puspitapallab Chaudhuri • H. O. Frota • \\ Cicero Mota • Angsula Ghosh
}

Received: 2 October 2017 / Accepted: 17 May 2018 / Published online: 8 June 2018

(C) Springer Science+Business Media B.V., part of Springer Nature 2018

\begin{abstract}
We report theoretical analysis of charge transport process through a single glycine molecule utilizing graphene nanogaps. Density functional theory and non-equilibrium Green's function method are employed to investigate the transport properties of glycine inside the gap. The projected density of states, transmittance, and the current-voltage characteristics are determined with changes in the molecular orientation inside the nanogap of c.a $0.8 \mathrm{~nm}$. The current
\end{abstract}

P. Chaudhuri $(\bowtie) \cdot$ H. O. Frota $\cdot$ A. Ghosh

Department of Physics, Federal University of Amazonas, Manaus, 69077-000, Amazonas Brazil

e-mail: puspito@ufam.edu.br

H. O. Frota

e-mail: hfrota@ufam.edu.br

A. Ghosh

e-mail: angsula@ufam.edu.br

C. Mota

Department of Mathematics, Federal University

of Amazonas, Manaus, 69077-000, Brazil

e-mail: cicmota@gmail.com

Present Address:

A. Ghosh

Institute of Physics, University of São Paulo, São Paulo, 05508-090, São Paulo Brazil

\section{Present Address:}

P. Chaudhuri

Institute for Theoretical Physics, São Paulo State University,

São Paulo, São Paulo Brazil values demonstrate a high sensitivity on the orientation of the molecule. The conductance of the molecule is also dependent on the voltage.

Keywords Glycine - Graphene nanogap · DFT · Molecular electronics · Nanoelectronics .

Modeling and simulation

\section{Introduction}

Currently, the conventional silicon ( $\mathrm{Si}$ )-based technology for electronic devices is facing enormous challenges due to the ever-increasing demand for miniaturization of the active components. The astounding progress in nanotechnology, in last few decades, is accelerating the process of downscaling and cost reduction of electronic devices paving the way for the entry of new concepts like 2D electronics (graphene) (Fiori et al. 2014; Cao et al. 2013; Wu et al. 2013), organic electronics (conducting polymers) (Rogers et al. 2010; Facchetti 2011), spintronics (Wolf et al. 2001; Tetienne et al. 2014), and molecular electronics (Aviram and Ratner 1974; Tour 2000; Sun et al. 2014). In order to scale down the devices below $20 \mathrm{~nm}$, investigations on the possibility of single molecule conductance are becoming important more than ever. No doubt, single-molecule electronics (molecular electronics) that involves the action of electrodes with a single molecule in between them represents the ultimate limit of miniaturization of electronic devices 
(Sun et al. 2014; Kima et al. 2014). However, largescale fabrication of fully functional single molecule electronic circuit is still far from reality, although some significant development has been achieved with respect to the construction of meta-molecule-metal junctions that includes the use of nanowires (Cobden 2001; Wang et al. 2014; Dasgupta et al. 2014), nanotubes (Sorgenfrei et al. 2011; Liu et al. 2010), nanogaps (Du et al. 2009; Yaghmaie et al. 2010), nanopores (Howorka and Siwy 2009; Arjmandi-Tash et al. 2016; Lagerqvist et al. 2006), mechanical break junctions (Xu et al. 2003; Zhao et al. 2014), mechanical cantilevers (Burg et al. 2007), electromigration (Park et al. 1999), electron beam lithography (Nicewarner-Pena et al. 2001; Qin et al. 2005), molecular rulers (Hatzor and Weiss 2001; Dadosh et al. 2005), scanning tunneling microscopy (STM), atomic force microscopy (AFM) (Xu et al. 2003; Sader et al. 2005), and others. Studying the single molecule electronic circuits is extremely important in understanding the transport behavior at a single-molecule level in order to prepare entirely molecular integrated circuits (Cui et al. 2015).

Graphene, with its novel electronic, thermal, mechanical, and chemical properties, has always been very promising with broad range of applications in Material Science and Engineering. It has widely been accepted as a propitious next-generation conducting material that can replace traditional electrode materials such as indium tin oxide in electrical and optical devices (Jo et al. 2010, 2012; Hong et al. 2013; Mol et al. 2015). Electrical characteristics of graphenebased devices have also drawn a lot of attention (Horri et al. 2017a, b, c) Presently, graphene is widely used in nanopore-nanogap devices. Recent advances have already been made in the fabrication of solidstate nanopores (Wu et al. 2009; Taniguchi et al. 2009) and their applications in whole-genome analysis (Lagerqvist et al. 2006; Storm et al. 2005; Zwolak and Di Ventra 2005; Iqbal et al. 2007; Dekker 2007). The solid-state nanopores have emerged as promising sensors due to their better stability and also due to its use both as membrane material as well as electrodes. It is an even more able device for DNA sequencing because of its extraordinary small thickness of graphene $(0.3 \mathrm{~nm})$ which is comparable to the DNA base pair stacking distance of $0.35 \mathrm{~nm}$ (Sathe et al. 2011). Graphene nanogaps have recently been successfully used to measure the tunneling current and detect translocation events in order to perform DNA sequencing (Postma 2010). The tunneling current is highly sensitive to the separation between the two electrodes and the size, shape, and orientation of the conducting molecules residing in the nanogap (Fanget et al. 2014). Furthermore, the conformational changes that the molecular structure may undergo can also affect transport behavior significantly (Galperin et al. 2007; Troisi and Ratner 2006; Gaudioso et al. 2000). Moreover, the conductance of the monolayer graphene nanopore was found to be higher than multilayer graphene nanopore. The structuring of the nanoribbon depends on the edge profile. Several studies demonstrate a semi-conducting behavior of the armchair ribbon compared to the metallic behavior of the zigzag-edged ribbon (Heerema and Dekker 2016).

Density functional studies in a graphene nanopore/ nanogap setup have been carried out to distinguish between the DNA nucleotides. The tunneling transport properties of the four nucleotides inside a graphene nanogap have been important to observe the fluctuation of the tunneling current with the change in the nucleotide as well as the orientation of the nucleotides (Prasongkit et al. 2011, 2013). In several graphene nanopore studies, the dangling bonds at the graphene edges were typically saturated with hydrogen (Prasongkit et al. 2011; Nelson et al. 2010; He et al. 2001; Avdoshenko et al. 2013) or nitrogen (Saha et al. 2012). Functional groups attached to graphene are also utilized for transport calculations (Prasongkit et al. 2013).

Organic materials and biomolecules are showing promising electrical properties and are finding their ways to various applications that range from biomedical equipment and sensors to home theaters and TV systems (Shinwari et al. 2010). The lower current and power operations along with its price benefits make these molecules ideal for usage. Moreover, the conductive properties of the biomolecules are also being exploited for designing therapeutic equipments for, e.g., the DNA hybridization sensors (Shinwari et al. 2007; Landheer et al. 2005, 2007; Deen et al. 2006). Recent interest in understanding the transport of charge through single molecules such as DNA and protein and other molecules like benzene-1,4dithiolate, redox active transition metal complexes have been due to their relevance not only in physiological reactions but also in molecular electronic applications (Lagerqvist et al. 2006; Zwolak and Di Ventra 
2005; He et al. 2007; Chang et al. 2010; Wang et al. 2007; Zhang et al. 2007; Haiss et al. 2007; Albrecht et al. 2005, 2006; Ivanov et al. 2011; Ventra et al. 2000; Reed et al. 1997; Schneider et al. 2010). The electrical conductance of DNA and proteins remains a subject of intense research. The electrical conductivities of the proteins should play a big role in their function as structural/transport units of enzymes. In fact, they may be important for use in electrodes, electronic devices, and sensors. The single-molecule DNA and protein sensors can be implemented for a faster investigation of the effects of inhibitory drugs through a study of the conductance current (Lagerqvist et al. 2006, 2007a, b; Zwolak and Di Ventra 2005, 2008; Zikic et al. 2006; Krems et al. 2009). Recently, the transport properties and the projected density of states of glycine molecule doped on carbon nanotube sandwiched between two carbon nanotube electrodes have been studied (Zhou et al. 2013). Glycine, with the molecular formula $\mathrm{NH}_{2} \mathrm{CH}_{2} \mathrm{COOH}$, as demonstrated in Fig. 1, is one of the most important biomolecules. It is the simplest and the smallest of all natural amino acids. Amino acids, as we all know, are the basic building blocks of proteins, which are formed through successive amide linkage (peptide bond) of several amino acids. Since glycine does not possess a side chain, it can easily adopt different conformations, giving high degree of local flexibility to the polypeptide. It occurs abundantly in certain fibrous proteins due to its flexibility and because of its small size it allows adjacent polypeptide chains to pack together closely (Yan et al. 1995). Although it contains just 10 atoms, glycine has all the essential characteristics of larger amino acids and peptide systems. Hence, it serves well as a model biomolecular system and has long been a subject of intense research, both experimentally and theoretically, to study the structural characteristics as well as the intra- and intermolecular interactions in biomolecular systems under different environmental situations.

In the present work, we use the first principles DFT method to study the transport properties of a single molecule of glycine inside a single-layer graphene nanogap with a zigzag edge. In spite of the fact that perfect armchair or zigzag edges cannot be realized thermodynamically (Girit et al. 2009; Ritter and Lyding 2009), the elucidation of the transport properties associated to a zigzag edge is important not only for graphene-edge applications but also for fundamental physics, chemistry, and nanoscience (Goto et al. 2013). The so-called edge state in zigzag edges due to the $p_{z}$ electrons confined on the outer carbon atoms distinguishes the above from the corresponding armchair versions (Nakada et al. 1996; Son et al. 2006; Acik and Chabal 2011). The graphene electrodes with zigzag edges have been chemically passivated by hydrogen. A short description of the computational procedure is presented in "Computational details". In "Results and discussion", the electronic properties are discussed in detail. Finally, a short summary and conclusion are given in "Conclusion".

\section{Computational details}

We study the tunneling properties along with the projected density of states (PDos) of the glycine molecule when located between the graphene electrodes with zigzag edges chemically passivated by hydrogen. The equilibrium geometry of the glycine molecule along with its electrostatic potential surface has been demonstrated in Fig. 1. The red color indicates the negative region whereas the blue indicates the positive one. The $\mathrm{O}_{4}$ and $\mathrm{N}_{1}$ atoms are the most electronegative ones
Fig. 1 a Isolated glycine molecule where the constituent atoms have been identified by atomic symbols and the $\mathbf{b}$ electrostatic potential surface of isolated glycine
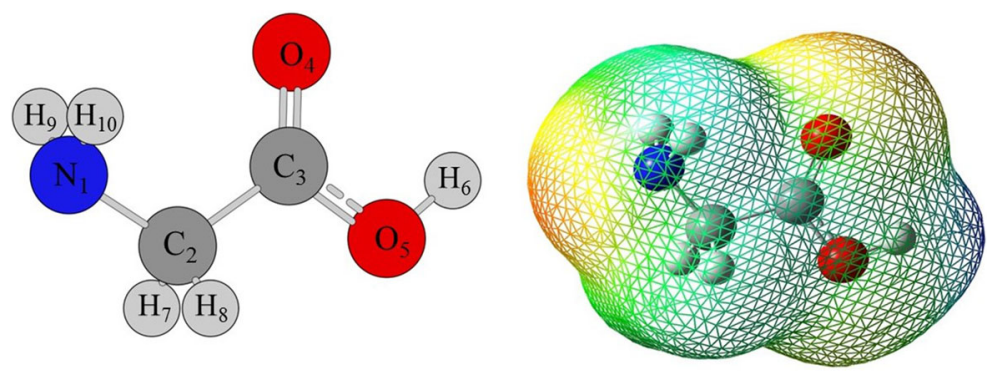

(a) (b) 
as can be seen from the figure. The system consists of three regions: the glycine molecule in the center of the two electrodes and the left and right graphene electrodes. This graphene-glycine-graphene system has been constructed, first by optimizing the isolated glycine molecule and the graphene electrodes separately and then placing the glycine molecule inside the nanogap of graphene as shown in Fig. 2. An electrode-electrode initial spacing of $7.9 \AA$ along the $z$-axis is considered (measured from $\mathrm{H}$ to $\mathrm{H}$ ). The glycine molecule is accommodated within this gap of graphene such that the extreme atoms of the glycine molecule are $\sim 2 \AA$ away from the nearest hydrogen atom of the graphene electrodes along the $z$-direction. The width of the graphene sheet is $9.6 \AA$ along the $z$ axis and $16 \AA$ along the $x$-axis in order to ensure that the perturbation effects from the glycine molecule are sufficiently screened. Periodic boundary conditions along the electrode edges effectively create repeated images of the glycine molecule separated by $13 \AA$, sufficiently large to avoid any sort of interaction between them. The combined system of graphene and glycine has been optimized allowing the atoms in the central region to relax. The glycine molecule is positioned to lie in the plane of the graphene electrodes as shown in Fig. 2. The position of the glycine molecule in the above figure is considered to be the initial orientation with $\theta=0^{\circ}$. The coordinate axes of the above system have also been shown in the figure. We also consider the effect of rotation on the density of states, transmittance, and current. The molecule is rotated in steps of $30^{\circ}$ around the $y$-axis from $0^{\circ}$ to $180^{\circ}$ and is translated so that the gap between left electrode glycine and right electrode glycine is always $\sim 2 \AA$. All optimizations are performed using the density-functional method as implemented in the

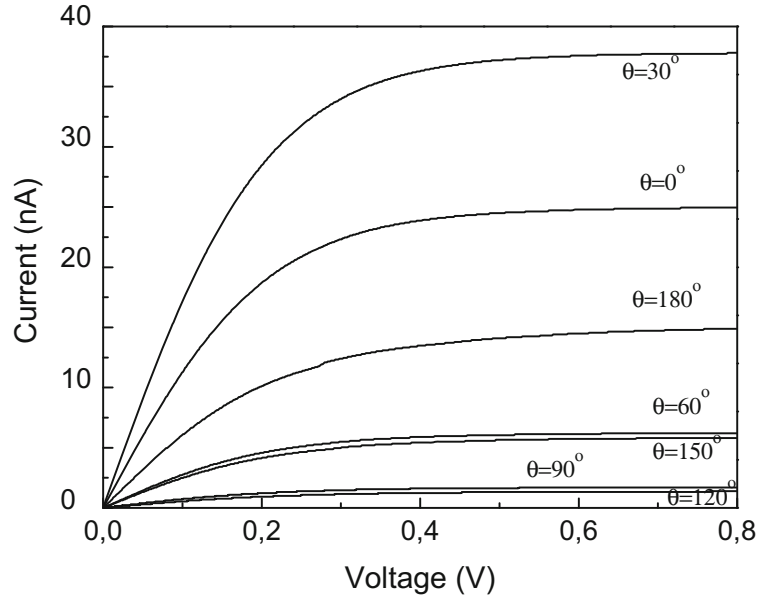

Fig. 3 Current-voltage curves for the glycine molecule at different orientations according to the legend in the figure

Quantum-Espresso package (Giannozzi et al. 2009). The BFGS quasi-Newton algorithm has been adopted for the constraint-free geometry optimization with the convergence thresholds set at $10^{-3} \mathrm{eV} / \AA$ for force and $10^{-4} \mathrm{eV}$ for energy. The real-space integration is performed using a 40 Ry cutoff, and due to the large cell size, all the optimizations have been done only at the $\Gamma$ point. The Brillouin zone of the supercell is sampled by $6 \times 1 \times 6$ Monkhorst-Pack $k$-point grid. The construction and visualization of the molecular structures are performed using the XCrySDen (Kokalj 2003) and Gaussview 4.1 (Dennington et al. 2006) packages. After the geometry optimization, the total and projected densities of states were obtained utilizing the Quantum-Espresso package.

The transport properties for each glycine orientation are calculated following the Landauer formula
Fig. 2 Illustration of the graphene nano-electrodes for measuring the conductance of single glycine molecule. The above orientation of the glycine molecule is at $\theta=0^{\circ}$

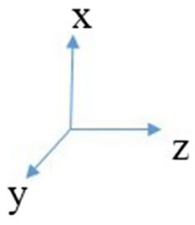




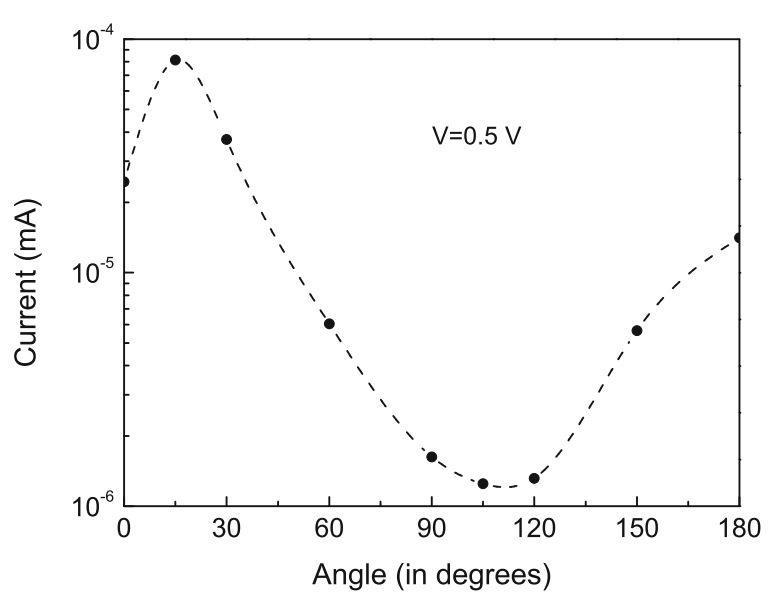

Fig. 4 Current dependencies of the glycine molecule on orientation for a bias voltage $\left(V_{\text {bias }}=0.5 \mathrm{eV}\right)$

as implemented in the WanT package for quantum transport (Calzolari et al. 2004; Ferretti et al. 2010). The technique combines the DFT and non-equilibrium Green's function method. The transmittance is calculated as a function of energy for the angles between $0^{\circ}$ and $180^{\circ}$ in steps of $30^{\circ}$. The current was calculated integrating the transmittance under an applied bias voltage. The chemical potentials related to the left and the right leads are given by $-0.5 \mathrm{~V}$ and $0.5 \mathrm{~V}$ respectively.

\section{Results and discussion}

The transport properties of the glycine molecule in the single-layer hydrogen-passivated graphene nanogap with zigzag edges are discussed in detail. The above system is found to be the most energetically favorable one when compared with the armchair graphene edges and also with that of a zigzag graphene layer of reduced width. The results on the I-V (currentvoltage) characteristics of the system, as represented in Fig. 2, are presented in Fig. 3 for the angles between $0^{\circ}$ and $180^{\circ}$ in steps of $30^{\circ}$. The maximum current is observed for $\theta=30^{\circ}$ for the entire range of the bias voltage whereas the minimum current is for $\theta=120^{\circ}$. The magnitudes of the current at different orientations can be ordered in the following manner : $I_{30}>$ $I_{0}>I_{180}>I_{60}>I_{150}>I_{90}>I_{120}$. The current through the graphene nanogap in the absence of the glycine molecule is few orders of magnitude less than that with the glycine. Furthermore, the absence of the glycine molecule yields a system that is energetically less favorable. Inclusion of glycine in the graphene nanogap shifts the Fermi energy upward by a considerable amount. Hence, the transport behavior is significantly modified. Moreover, it is also much higher than that through the nucleotides (Prasongkit et al. 2011) within the graphene nanogap passivated by hydrogen atoms and even with the functional groups (Prasongkit et al. 2013). However, the current of carbon nanotube
Fig. 5 Projected densities of states (PDos) of the a hydrogen ( $H_{6}$ (purple), $H_{7}$ (green), $H_{8}$ (cyan), $H_{9}$ (red), $H_{10}$ (black)), b oxygen $\left(\mathrm{O}_{5}(1 \mathrm{~s}\right.$-red, $2 p$-purple), $\mathrm{O}_{4}(1 s$ black, $2 p$-green)), c nitrogen $\left(N_{1}\right.$ (1s-black, $2 p$-green)), and $\mathbf{d}$ carbon atoms $\left(C_{2}\right.$ (1s-black, $2 p$-green), $C_{3}$ (1s-red, $2 p$-purple)) of the molecule for $\theta=0^{\circ}$
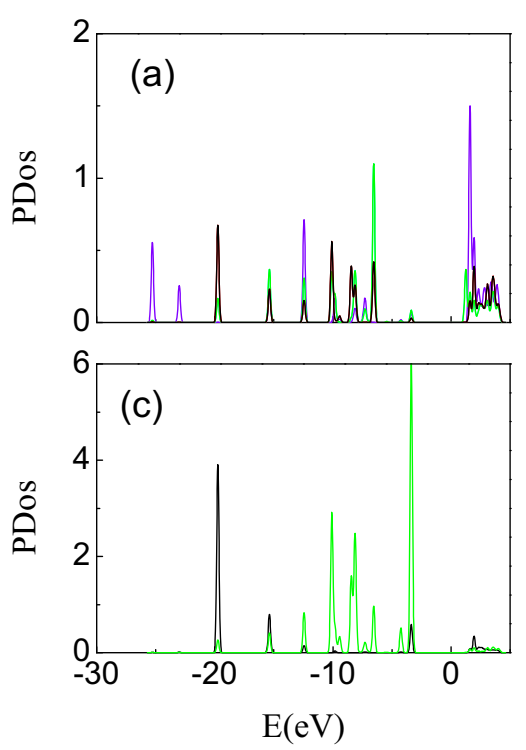
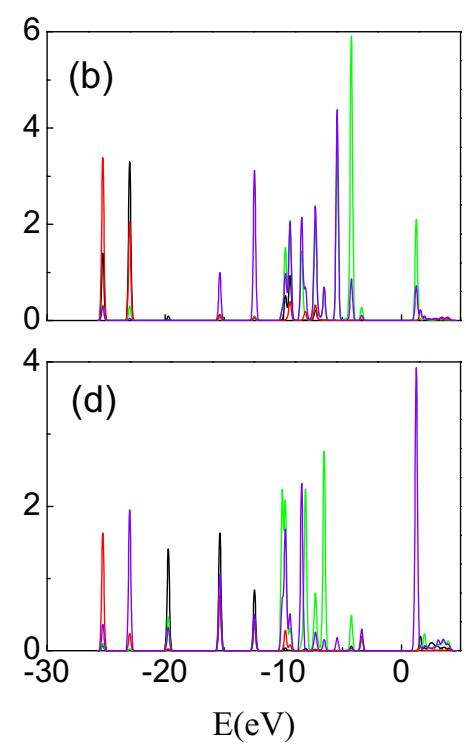
Fig. 6 Projected densities of states (PDos) of the a hydrogen $\left(H_{6}\right.$ (purple), $\mathrm{H}_{7}$ (green), $\mathrm{H}_{8}$ (cyan), $\mathrm{H}_{9}$ (red), $H_{10}$ (black)), b oxygen $\left(O_{5}(1 s\right.$-red, $2 p$-purple), $O_{4}$ ( $1 s$-black, $2 p$-green $))$, c nitrogen $\left(N_{1}\right.$ (1s-black, $2 p$-green $)$, and d carbon atoms $\left(C_{2}\right.$ (1s-black, $2 p$-green), $C_{3}$ (1s-red, $2 p$-purple)) of the glycine molecule for $\theta=30^{\circ}$
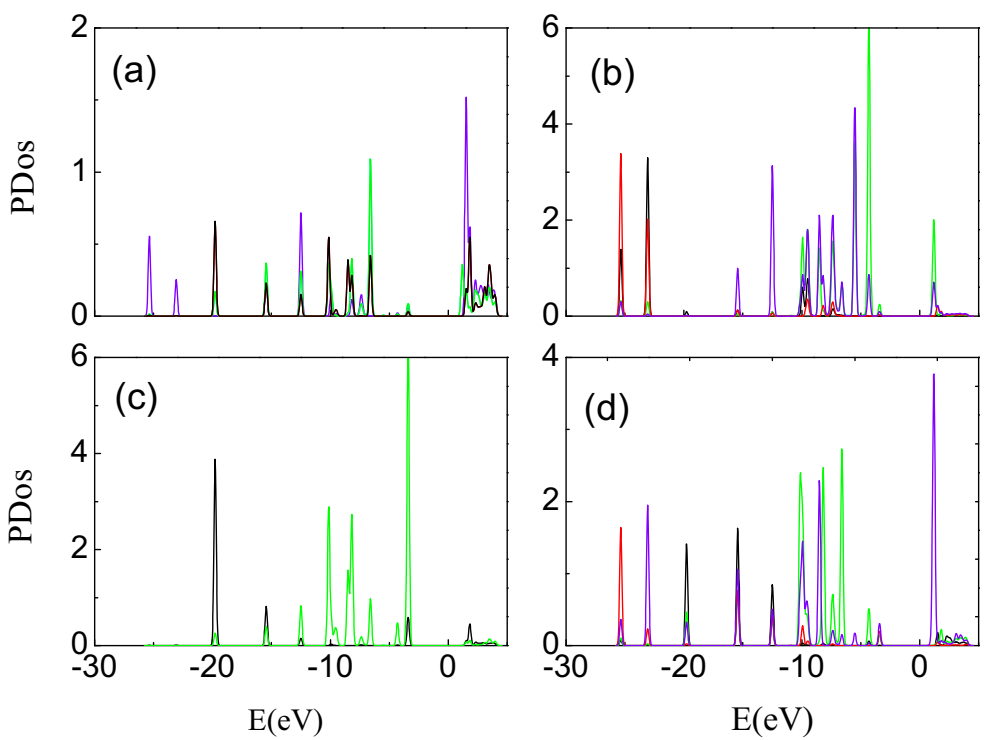

doped with glycine (Zhou et al. 2013) is higher when compared to our results. The effect of the width of the graphene layer and also the nanogap width are considered. The current decreases with the increase in nanogap width as was also observed in Postma (2010). The current was found to be sensitive to the width of the graphene monolayer. A slight increase in the current was observed with the increase in the width of the graphene leads. The edge profile of the leads were also investigated. The armchair leads passivated with hydrogen leads to a higher current than the zigzag one.
Figure 4 demonstrates the current vs the angle of rotation for a value of bias voltage of $V_{\text {bias }}=0.5 \mathrm{eV}$. A dotted line as a guide to the eye has been plotted in order to help in the visualization of its dependence. A change of nearly one order of magnitude has been observed among the different orientations. The current varies between nearly $80 \mathrm{nA}$ for $\theta=15^{\circ}$ and $1.3 \mathrm{nA}$ for $\theta=105^{\circ}$ at $V_{\text {bias }}=0.5 \mathrm{eV}$. Hence, the conductivity depends highly on the orientation of the molecule in the nanogap. The conductivity increases considerably as we change the orientation of the molecule.
Fig. 7 Projected densities of states (PDos) of the a hydrogen ( $H_{6}$ (purple), $H_{7}$ (green), $H_{8}$ (cyan), $H_{9}$ (red), $H_{10}$ (black)), b oxygen $\left(\mathrm{O}_{5}(1 s\right.$-red, $2 p$-purple), $O_{4}$ ( $1 s$-black, $2 p$-green $)$, c nitrogen $\left(N_{1}\right.$ (1s-black, $2 p$-green)), and d carbon atoms $\left(C_{2}\right.$ (1s-black, $2 p$-green), $C_{3}$ ( $1 s$-red, $2 p$-purple)) of the glycine molecule for $\theta=60^{\circ}$
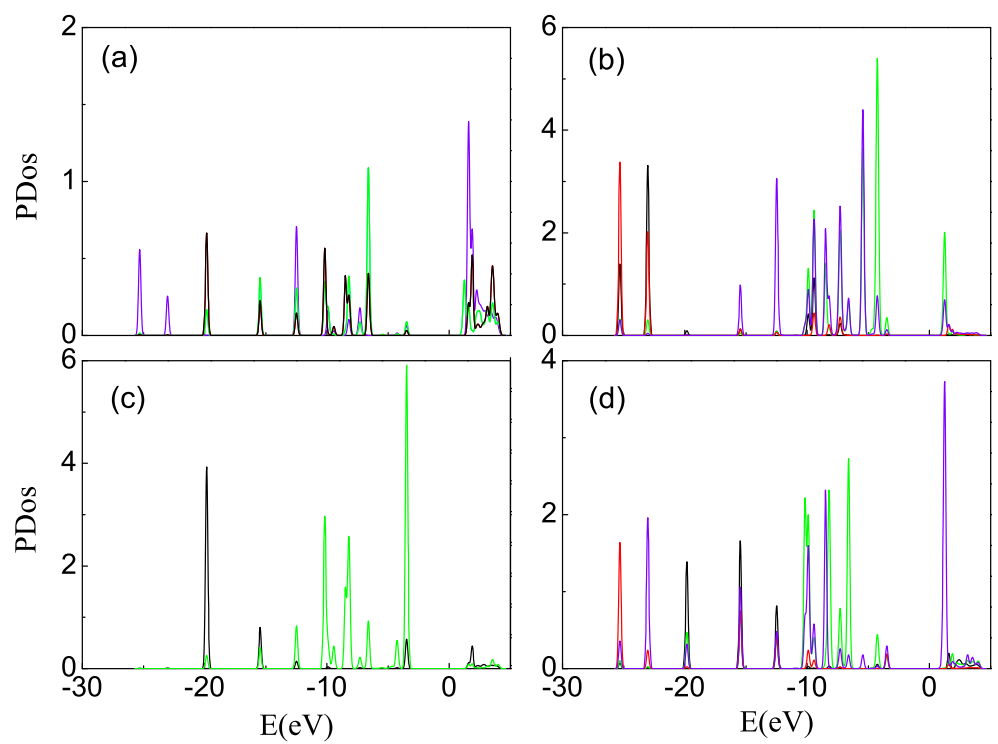
The position and the orientation of the $\mathrm{O}_{4}$ and $N_{1}$ atoms are of utmost importance for the current. The PDos peaks of the $2 p$ orbitals of the $O_{4}$ and $N_{1}$ atoms corroborate with the above fact. The high electronegativity of the $\mathrm{O}_{4}$ and $\mathrm{N}_{1}$ atoms observed in the electrostatic potential surface of glycine also indicates higher participation of the above atoms. Moreover, we see that the peaks of the $2 p$ orbitals of $\mathrm{O}_{4}$ and $N_{1}$, the transmittance value, and the total density of states below the Fermi energy are associated with the HOMO of the isolated glycine molecule. When the molecule is rotated, the peak magnitude changes and also the peak position suffers changes relative to the Fermi energy and thus the current is affected. Moreover, the current drops by increasing the distance between the glycine molecule and the graphene leads.

In Figs. 5, 6, 7, and 8, PDos for all the atoms of the glycine molecule for different configurations are presented. The PDos of the five hydrogen atoms are plotted for $\theta=0^{\circ}, 30^{\circ}, 60^{\circ}$, and $180^{\circ}$ in Figs. 5a, 6a, 7a, and $8 \mathrm{a}$ respectively. The contribution of two hydrogen atoms $\left(H_{7}\right.$ and $\left.H_{8}\right)$ linked to the carbon atom is represented in cyan and green, whereas those bonded to the nitrogen atoms $\left(H_{9}\right.$ and $\left.H_{10}\right)$ are plotted in red and black respectively. The PDos of $H_{6}$ demonstrated in violet have the largest contribution. The PDos of the oxygen atoms $O_{4}$ and $O_{5}$ (carbon atoms $C_{1}$ and $C_{2}$ ) are plotted in Figs. 5b, d, 6b, d, 7b, d, and 8b, d. The PDos of the nitrogen atom are plotted in Figs. $5 c, 6 c, 7 c$, and $8 c$.

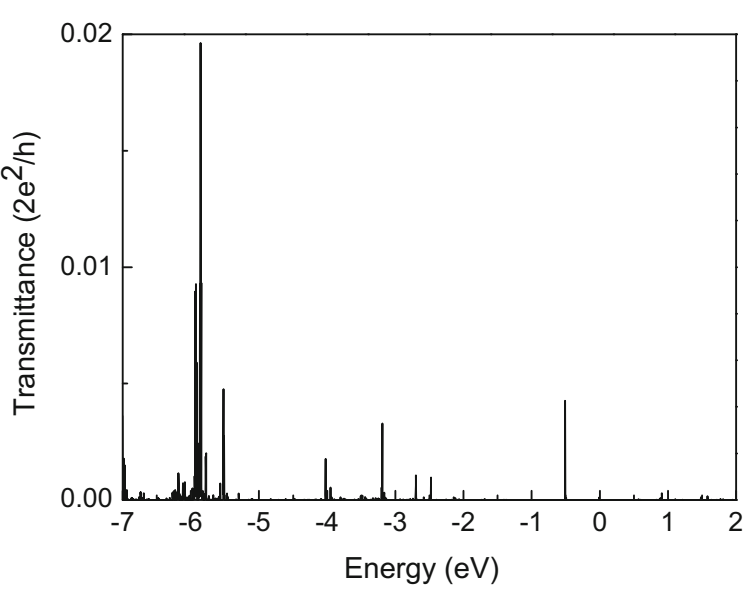

Fig. 9 Transmittance as a function of the energy $(E)$, for $\theta=0^{\circ}$

In all the above five atoms $\left(O_{4}, O_{5}, C_{2}, C_{3}, N_{1}\right)$, the contribution of the $2 p$ orbital to the density of states predominates throughout the considered energy range. The $s$ orbital also exhibits a significant contribution at low energies. However, it is interesting to note the dependence of the atomic PDos on the angle of rotation. While at $\theta=0^{\circ}, H_{8}$ and $H_{9}$ have a higher contribution, at $\theta=30^{\circ}, H_{10}$ and $H_{7}$ have larger DOS. The contribution from $H_{6}$ continues to demonstrate very little alteration with rotation and has the highest DOS at high energies. The highest contributions to the PDos are from the oxygen $\left(\mathrm{O}_{4}\right)$ atom and the nitrogen $\left(N_{1}\right)$ atom of the glycine molecule for all
Fig. 8 Projected densities of states (PDos) of the a hydrogen $\left(H_{6}\right.$ (purple), $H_{7}$ (green), $H_{8}$ (cyan), $H_{9}$ (red), $H_{10}$ (black)), b oxygen $\left(O_{5}(1 s\right.$-red, $2 p$-purple), $O_{4}(1 s$-black, $2 p$-green $))$, c nitrogen $\left(N_{1}\right.$ (1s-black, $2 p$-green)), and d carbon atoms $\left(C_{2}\right.$ (1s-black, $2 p$-green), $C_{3}$ (1s-red, $2 p$-purple)) of the glycine molecule for $\theta=180^{\circ}$
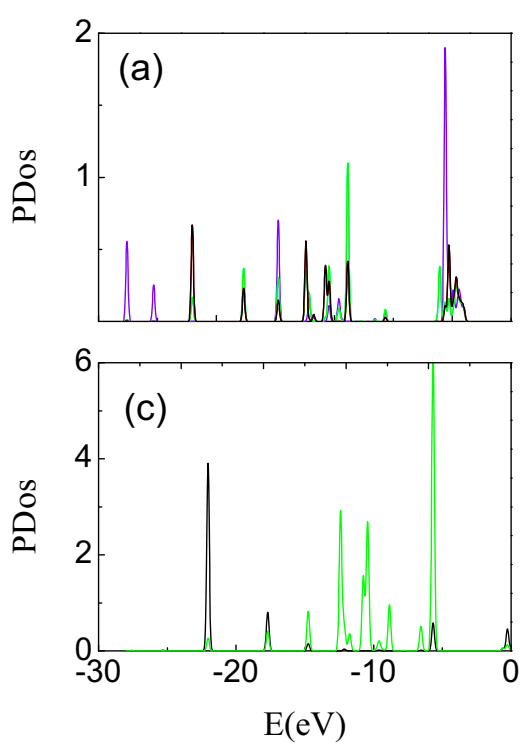
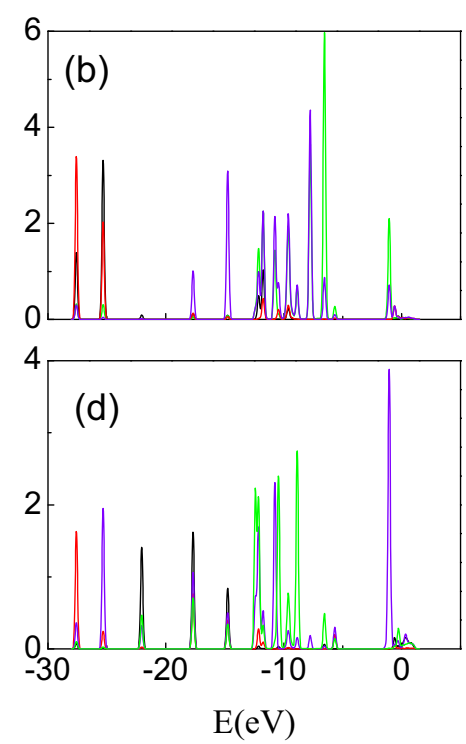
Fig. 10 Transmittance as a function of the energy $(E)$, for $\mathbf{a} \theta=30^{\circ}, \mathbf{b} \theta=60^{\circ}$, $\mathbf{c} \theta=90^{\circ}, \mathbf{d} \theta=120^{\circ}$, e $\theta=150^{\circ}, \mathbf{f} \theta=180^{\circ}$

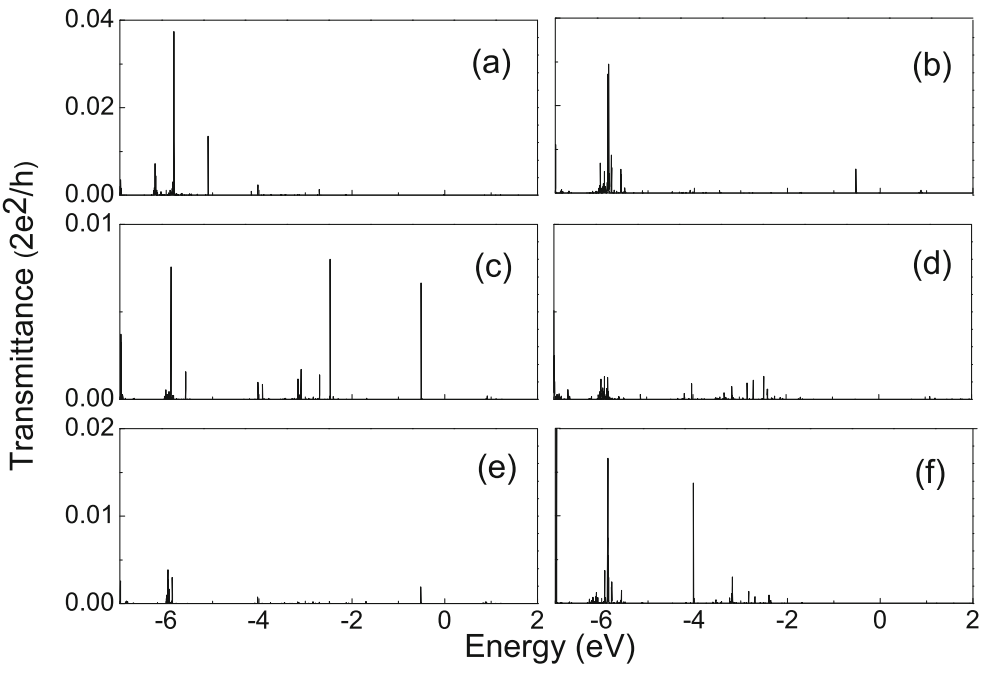

the angles in agreement with electronic nature of the molecule as seen in Fig. 1. The $2 p$ contributions are most prominent for the above atoms and the peaks can be associated with the HOMO of the isolated glycine molecule. The highest contribution from the carbon atom $\left(C_{3}\right)$ and hydrogen atom $\left(H_{6}\right)$ could be associated with the LUMO of the isolated glycine. Moreover, the $2 p$ contribution of the $O_{4}$ atom is highest for $\theta=30^{\circ}$ which decreases with the change in the orientation of the glycine molecule and accompanies the same behavior as observed in Fig. 4. From Figs. 5-8, we observe that the contribution of the $2 p$ orbital of $\mathrm{O}_{4}$ atom obeys the following order : $\operatorname{PDos}_{30^{\circ}}>$ $P \operatorname{Dos}_{0^{\circ}}>P \operatorname{Dos}_{180^{\circ}}>P \operatorname{Dos}_{60^{\circ}}$. The total DOS also depends on the orientation of the glycine molecule between the hydrogenated graphene leads. Moreover, it is also associated with the HOMO of the isolated glycine.

The quantum conductance as a function of energy is shown in Fig. 9 for $\theta=0^{\circ}$. In Fig. 10a-f, the conductance values are given for (a) $\theta=30^{\circ}$, (b) $\theta=180^{\circ}$, (c) $\theta=90^{\circ}$, (d) $\theta=120^{\circ}$, (e) $\theta=150^{\circ}$, and (f) $\theta=60^{\circ}$ respectively.

Transmittance peaks below the Fermi energy are related to the peaks of the DOS which in turn are associated with the HOMO values of the isolated glycine. Moreover, the distance of the $O_{4}$ and $N_{1}$ atoms from the hydrogenated graphene sheet is important for the understanding of the change of the transmittance with orientation. The $2 p$ orbital peak of $\mathrm{O}_{4}$ atom also corroborates with the above fact. For example at $\theta=$ $30^{\circ}$, the nearest distance of $O_{4}$ from the leads is 2.26 $\AA$ compared to $4.25 \AA$ at $\theta=120^{\circ}$. This result is in accordance with the current as a function of bias voltage shown in Fig. 3.

\section{Conclusion}

In conclusion, the calculation demonstrates a potential use of the glycine molecule, the simplest and the smallest of all natural amino acids, in nanoscale electronics. The sensitive dependence of the tunneling current on the orientation of the glycine molecule inside the graphene nanogap can be useful for various therapeutic equipments. The preferred orientation of glycine that leads to the highest conductivity of the system is $\theta=15^{\circ}$, whereas the least favorable is one $\theta=105^{\circ}$. The projected density of states and the conductance calculations are also at par with the above findings.

Funding information This study received financial support from the Brazilian funding agency $\mathrm{CNPq}$.

\section{Compliance with ethical standards}

Conflict of interest The authors declare that they have no conflict of interest. 


\section{References}

Acik M, Chabal YJ (2011) Nature of graphene edges. Jpn J Appl Phys 50:070101

Albrecht T, Guckian A, Kuznetsov AM, Vos JG, Ulstrup JJ (2006) Mechanism of electrochemical charge transport in individual transition metal complexes. J Am Chem Soc 128:17132-17138

Albrecht T, Guckian A, Ulstrup J, Vos JG (2005) Transistor-like behavior of transition metal complexes. Nano Lett 5:14511455

Arjmandi-Tash H, Belyaeva LA, Schneider GF (2016) Single molecule detection with graphene and other twodimensional materials: nanopores and beyond. Chem Soc Rev 45:476-493

Avdoshenko SM, Nozaki D, da Rocha CG, González JW, Lee MH, Gutierrez R, Cuniberti G (2013) Dynamic and electronic transport properties of DNA translocation through graphene. Nanopores Nano Lett 13:1969-1976

Aviram A, Ratner MA (1974) Molecular rectifiers. Chem Phys Lett 29:277-283

Burg TP, Godin M, Knudsen SM, Shen W, Carlson G, Foster JS, Babcock K, Manalis SR (2007) Weighing of biomolecules, single cells and single nanoparticles in fluid. Nature 446:1066-1069

Calzolari A, Marzari N Souza I, Nardelli MB (2004) Ab initio transport properties of nanostructures from maximally localized Wannier functions. Phys. Rev B 69:035108

Cao W, Kang J, Liu W, Khatami Y, Sarkar D, Banerjee K (2013) 2D electronics: graphene and beyond. In: Proc Eur Solid-State Device Res Conf, pp 37-44

Chang S, Huang S, He J, Liang F, Zhang P, Li S, Chen X, Sankey O, Lindsay S (2010) Electronic signatures of all four DNA nucleosides in a tunneling gap. Nano Lett 10:1070-1075

Cobden DH (2001) Molecular electronics: nanowires begin to shine. Nature 409:32-33

Cui A, Dong H, Hu W (2015) Nanogap electrodes towards solid state single-molecule transistors. Small 11:6115-6141

Dadosh T, Gordin Y, Krahne R, Khivrich I, Mahalu D, Frydman V, Sperling J, Yacoby A, Bar-Joseph I (2005) Measurement of the conductance of single conjugated molecules. Nature 436:677-680

Dasgupta NP, Sun J, Liu C, Brittman S, Andrews SC, Lim J, Gao H, Yan R, Yang P (2014) 25th anniversary article: semiconductor nanowires-synthesis, characterization, and applications. Adv Mater 26:2137-2184

Deen MJ, Shinwari MW, Ranuárez JC, Landheer D (2006) Noise considerations in field-effect biosensors. J Appl Phys 100:074703-074703-8

Dekker C (2007) Solid-state nanopores. Nat Nanotechnol 2:209-215

Dennington R, Keith T, Millam J (2006) GaussView, Version 4.1.2. Semichem Inc., Shawnee Mission

Du K, Knutson CR, Glogowski E, McCarthy KD, Shenhar R, Rotello VM, Tuominen MT, Emrick T, Russell TP, Dinsmore AD (2009) Self-assembled electrical contact to nanoparticles using metallic droplets. Small 5:19741977
Facchetti A (2011) $\pi$-conjugated polymers for organic electronics and photovoltaic cell applications. Chem Mater 223:733-758

Fanget A, Traversi F, Khlybov S, Granjon P, Magrez A, Forro L, Radenovic A (2014) Nanopore integrated nanogaps for DNA detection. Nano Lett 14:244-249

Ferretti F, Bonferroni B, Calzolari A, Buongiorno MN (2010) WanT Code, http://www.wannier-transport.org

Fiori G, Bonaccorso F, Lannaccone G, Palacios T, Neumaier D, Seabaugh A, Banerjee SK, Colombo L (2014) Electronics based on two-dimensional materials. Nat Nanotech 9:768779

Gaudioso J, Lauhon LJ, Ho W (2000) Vibrationally mediated negative differential resistance in a single molecule. Phys Rev Lett 85:1918-1921

Galperin M, Ratner MA, Nitzan A (2007) Molecular transport junctions: vibrational effects. J Phys: Condens Matter 103201:19

Giannozzi P et al (2009) QUANTUM ESPRESSO: a modular and open-source software project for quantum simulations of materials. J Phys Condens Matter 21:1-19

Girit CO, Meyer JC, Emi R, Rossell MD, Kisielowski C, Yang L, Park C-H, Crommie MF, Cohen ML, Louie SG, Zettl A (2009) Controlled formation of sharp zigzag and armchair edges in graphitic nanoribbons. Science 323:1705-1708

Goto H, Uesugi E, Eguchi R, Fujiwara A, Kubozono Y (2013) Edge-dependent transport properties in graphene. Nano Lett 13:1126-1130

Haiss W, Albrecht T, van Zalinge H, Higgins SL, Bethell D, Höbenreich H, Schiffrin DJ, Nichols RJ, Kuznetsov AM, Zhang J, Chi Q, Ulstrup J (2007) Single molecule condutance of redox molecules in electrochemical scanning tunneling microscopy. J Phys Chem B 111:6703-6712

Hatzor A, Weiss PS (2001) Molecular rulers for scaling down nanostructures. Science 291:1019-1020

He J, Lin L, Zhang P, Lindsay S (2007) Identification of DNA basepairing via tunnel-current decay. Nano Lett 7:38543858

He YH, Scheicher RH, Grigoriev A, Ahuja R, Long SB, Huo ZL, Liu M (2001) Enhanced DNA sequencing performance through edge-hydrogenation of graphene electrodes. Adv Funct Mater 21:2674-2679

Heerema SJ, Dekker C (2016) Graphene nanodevices for DNA sequencing. Nat Nanotechnol 11:127-134

Hong G, Wu Q-H, Ren J, Wang C, Zhang W, Lee S-T (2013) Recent progress in organic molecule/graphene interfaces. Nano Today 8:388-402

Horri A, Faez R, Pourfath M, Darvish G (2017a) A computational study of vertical tunneling transistors based on graphene-WS $\mathrm{W}_{2}$ heterostructure. J Appl Phys 121:214503

Horri A, Faez R, Pourfath M, Darvish G (2017b) Modelling of a vertical tunneling transistors based on graphene- $\mathrm{WS}_{2}$ heterostructure. IEEE Trans Elect Dev 99:1-7

Horri A, Faez R, Darvish G (2017c) Numerical simulation of vertical tunneling transistor with bilayer graphene as source and drain regions. Phys Sta Solidi A 214:1700155

Howorka S, Siwy Z (2009) Nanopore analytics: sensing of single molecules. Chem Soc Rev 38:2360-2384

Iqbal SM, Akin D, Bashir R (2007) Solid-state nanopore channels with DNA selectivity. Nat Nanotechnol 2:243-248 
Ivanov AP, Instuli E, McGilvery CM, Baldwin G, McComb DW, Albrecht T, Ede JB (2011) DNA tunneling detector embedded in a nanopore. Nano Lett 11:279-285

Jo G, Choe M, Cho CY, Kim JH, Park W, Lee S, Hong WK, Kim TW, Park SJ, Hong BH, Kahng YH, Lee T (2010) Large-scale patterned multi-layer graphene films as transparent conducting electrodes for GaN light-emitting diodes. Nanotechnology 21:175201-175206

Jo G, Choe M, Lee S, Park W, Kahng YH, Lee T (2012) The application of graphene as electrodes in electrical and optical devices. Nanotechnology 23:112001

Kima T, Liu Z-F, Lee C, Neaton JB, Venkataramana L (2014) Charge transport and rectification in molecular junctions formed with carbon-based electrodes. PNAS 111:1092810932

Kokalj A (2003) Computer graphics and graphical user interfaces as tools in simulations of matter at the atomic scale. Compl Mat Sci 28:155-168

Krems M, Zwolak M, Pershin YV, Di Ventra M (2009) Effect of noise on DNA sequencing via transverse electronic transport. Biophys J 97:1990-1996

Lagerqvist J, Zwolak M, Ventra M (2006) Fast DNA sequencing via transverse electronic transport. Nano Lett 6:779-782

Lagerqvist J, Zwolak M, Di Ventra M (2007a) Comment on characterization of the tunneling conductance across DNA bases. Phys Rev E 76:013901/1-3

Lagerqvist J, Zwolak M, Ventra M (2007b) Influence of the environment and probes on rapid DNA sequencing via transverse electronic transport. Biophys J 93:2384-2390

Landheer D, Aers G, McKinnon WR, Deen MJ, Ranuarez JC (2005) Model for the field effect from layers of biological macromolecules on the gates of metal-oxide-semiconductor transistors. J Appl Phys 98:044701/1-15

Landheer D, McKinnon WR, Aers G, Jiang W, Deen MJ, Shinwari MW (2007) Calculation of the response of field-effect transistors to charged biological molecules. IEEE Sens J 7:1233-1242

Liu H, He J, Tang J, Liu H, Pang P, Cao D, Krstic P, Joseph S, Lindsay S, Nuckolls C (2010) Translocation of singlestranded DNA through single-walled carbon nanotubes. Science 327:64-67

Mol JA, Lau CS, Lewis WJM, Sadeghi H, Roche C, Cnossen A Warner JH, Lambert CJ, Anderson HL, Briggs GAD (2015) Graphene-porphyrin single-molecule transistors. Nanoscale 7:13181-13185

Nakada K, Fujita M, Dresselhaus MS, Dresselhaus MM (1996) Edge state in graphene ribbons: nanometer size effect and edge shape dependence. Phys Rev B 54:17954-17961

Nelson T, Zhang B, Prezhdo OV (2010) Detection of nucleic acids with graphene nanopores: ab initio characterization of a novel sequencing device. Nano Lett 10:3237-3242

Nicewarner-Pena SR, Freeman RG, Reiss BD, He L, Pena DJ, Walton ID, Cromer R, Keating CD, Natan MJ (2001) Submicrometer metallic barcodes. Science 294:137-141

Park H, Lim AKL, Alivisatos AP, Park J, McEuen PL (1999) Fabrication of metallic electrodes with nanometer separation by electromigration. Appl Phys Lett 75:301-303

Postma HWCh (2010) Rapid sequencing of individual DNA molecules in graphene nanogaps. Nano Lett 10:420-425

Prasongkit J, Grigoriev A, Pathak B, Ahuja R, Scheicher RH (2011) Transverse conductance of dna nucleotides in a graphene nanogap from first principles. Nano Lett 11:1941-1945

Prasongkit J, Grigoriev A, Pathak B, Ahuja R, Scheicher RH (2013) Theoretical study of electronic transport through DNA nucleotides in a double-functionalized graphene nanogap. J Phys Chem C 117:15421-15428

Qin L, Park S, Huang L, Mirkin CA (2005) On-wire lithography. Science 309:113-115

Reed MA, Zhou C, Muller CJ, Burgin TP, Tour JM (1997) Conductance of a molecular junction. Science 278:252-254

Ritter KA, Lyding JW (2009) The influence of edge structure on the electronic properties of graphene quantum dots and nanoribbons. Nat Mater 8:235-242

Rogers JA, Somya T, Huang Y (2010) Materials and mechanics for stretchable electronics. Science 327:1603-1607

Sader JE, Uchihashi T, Higgins MJ, Farrell A, Nakayama Y, Jarvis SP (2005) Quantitative force measurements using frequency modulation atomic force microscopytheoretical foundations. Nanotechnology 16:S94-S101

Saha KK, Drndić M, Nikolić BK (2012) DNA base-specific modulation of a transverse edge currents through a metallic graphene nanoribbon with a nanopore. Nano Lett 12:50-55

Sathe C, Zou X, Leburton J-P, Schulten K (2011) Computational investigation of DNA detection using graphene nanopores. ACS Nano 5:8842-8851

Schneider GF, Kowalczyk SW, Calado VE, Pandraud G, Zandbergen H, Vandersypen LMK, Dekker C (2010) DNA translocation through graphene nanopores. Nano Lett 630 10:3163-3167

Shinwari MW, Deen MJ, Landheer D (2007) Study of the electrolyte-insulator-semiconductor field-effect transistor (EISFET) with applications in biosensor design. Microelectron Reliab 47:2025-2057

Shinwari MW, Deen MJ, Starikov EB, Cuniberti G (2010) Electrical conductance in biological molecules. Adv Func Mater 20:1865-1883

Son Y-W, Cohen ML, Louie SG (2006) Energy gaps in graphene nanoribbons. Phys Rev Lett 97:216803

Sorgenfrei S, Chiu C-y, Gonzalez Jr RL, Yu Y-J, Kim P, Nuckolls C, Shepard KL (2011) Label-free single-molecule detection of DNA-hybridization kinetics with a carbon nanotube field-effect transistor. Nat Nanotechnol 6:126132

Storm AJ, Storm C, Chen JH, Zanderbergen H, Joanny JF, Dekker C (2005) Fast DNA translocation through a solidstate nanopore. Nano Lett 5:1193-1197

Sun L, Diaz-Fernandez YA, Gschneidtner TA, Westerlund F, Lara-Avilab S, Moth-Poulsen K (2014) Single-molecule electronics: from chemical design to functional devices. Chem Soc Rev 43:7378-7411

Taniguchi M, Tsutsui M, Yokota K, Kawai T (2009) Fabrication of the gating nanopore device. Appl Phys Lett 95:123701123703

Tetienne JP, THingant T, Kim JV, Diez LH, Adam JP, Garcia K, Roch JF, Rohart S, Thiaville A, Ravelosona D, Jacques V (2014) Nanoscale imaging and control of domain-wall hopping with a nitrogen-vacancy center microscope. Science 344:1366-1369

Tour JM (2000) Molecular electronics. Synthesis and testing of components. Acc Chem Res 33:791-804 
Troisi A, Ratner MA (2006) Molecular signatures in the transport properties of molecular wire junctions: what makes a junction molecular Small 2:172-181

Ventra MD, Pantelides ST, Lang ND (2000) First-principles calculation of transport properties of a molecular device. Phys Rev Lett 84:979-982

Wang J, Shen F, Wang Z, He G, Qin J, Cheng N, Yao M, Li L, Guo X (2014) Point decoration of silicon nanowires: an approach toward single-molecule electrical detection. Angew Chem 53:5038-5043

Wang W, Lee T, Reed MA (2007) Electrical characterization of self-assembled monolayers. In: Lyshevski SE (ed) Nano and molecular electronics handbook. CRC Press, Boca Raton. Chapter 1, pp 3

Wolf SA, Awschalom DD, Buhrman RA, Daughton JM, Molnar von S, Roukes ML, Chtchelkanova AY, Treger DM (2001) Spintronics: a spin-based electronics vision for the future. Science 294:1488-1495

Wu M-Y, Smeets RMM, Zandbergen M, Ziese U, Krapf D, Batson PE, Dekker NH, Dekker C, Zandbergen HW (2009) Control of shape and material composition of solid-state nanopores. Nano Lett 9:479-484

Wu Y, Farmer DB, Xia F, Avouris P (2013) Graphene electronics, materials, devices, and circuits. Proc IEEE 101:16201637

Xu BQ, Xiao XY, Tao NJ (2003) Measurements of singlemolecule electromechanical properties. J Am Chem Soc 125:16164-16165
Yaghmaie F, Fleck J, Gusman A, Prohaska R (2010) Improvement of PMMA electron-beam lithography performance in metal liftoff through a poly-imide bi-layer system. Microelectron Eng 87:2629-2632

Yan Y, Erickson BW, Tropsha A (1995) Free energies for folding and refolding of four types of .beta. Turns: simulation of the role of D/L chirality. J Amer Chem Soc 117:7592-7593

Zhao Y, Ashcroft B, Zhang P, Liu H, Sen S, Song W, Im J, Gyarfas B, Manna S, Biswas S, Borges C, Lindsay S (2014) Single molecule spectroscopy of amino acids and peptides by recognition tunneling. Nat Nanotechnol 9:466-473

Zhang J, Kuznetsov AM, Medvedev IG, Chi Q, Albrecht T, Jensen PS, Mertens SFL, Ulstrup J (2007) Intrinsic multistate switching of gold clusters through electrochemical gating. J Am Chem Soc 129:9162-9167

Zhou Y-h, Y-l Peng, Yuan L-z (2013) World congress on medical physics and biomedical engineering. IFMBE Proc 39:317

Zikic R, Krstic PS, Zhang X-G, Fuentes-Cabrera M, Wells J, Zhao X (2006) Characterization of the tunneling conductance across DNA bases. Phys Rev E 74:011919/1-9

Zwolak M, Di Ventra M (2005) Electronic signature of DNA nucleotides via transverse transport. Nano Lett 5:421-424

Zwolak M, Di Ventra M (2008) Colloquium: physical approaches to DNA sequencing and detection. Rev Mod Phys 80:141-165 